\title{
ANTI FUZZY BI-IDEALS ON ORDERED AG-GROUPOIDS
}

\author{
Nasreen Kausar ${ }^{1}$, Mohammad MuniR ${ }^{2}$, Muhammad Gulzar ${ }^{3}$, \\ Gezahagne Mulat Addis ${ }^{4}$, Rukhshanda Anjum ${ }^{5}$, \\ ${ }^{1}$ Department of Mathematics and Statistics, University of Agriculture, \\ Faisalabad, Pakistan, kausar.nasreen57@gmail.com \\ ${ }^{2}$ Department of Mathematics, Government Postgraduate College, \\ Abbottabad, Pakistan, dr.mohammadmunir@gpgc-atd.edu.pk \\ ${ }^{3}$ Department of Mathematics, Government College University Faisalabad, \\ Pakistan, 98kohly@gmail.com \\ ${ }^{4}$ Department of Mathematics, University of Gondar, Gondar, Ethiopia, \\ gezahagne412@gmail.com \\ ${ }^{5}$ Department of Mathematics and Statistics, University of Lahore, Lahore, \\ Pakistan, rukhshanda.anjum@math.uol.edu.pk
}

\begin{abstract}
The purpose of this study is to initiate the concept of anti fuzzy left (resp. right, bi-, generalized bi-, (1,2)-) ideals in ordered AG-groupoids. We characterize the different classes of ordered AG-groupoids in terms of such ideals. Particularl, we prove that in regular (resp. left weakly regular) ordered AG-groupoids, the concept of anti fuzzy (right, two-sided) ideals coincides.

Keywords: Anti fuzzy AG-subgroupoids, anti fuzzy left (resp. right, bi-, generalized bi-, (1,2)-) ideals. :
\end{abstract}

\section{INTRODUCTION}

In 1972, a generalization of abelian semigroups has been recognized by Kazim et. al [12]. In ternary commutative law: $a b c=c b a$, they introduced the braces on the left side of this law and initiated a new pseudo associative law, that is $(a b) c=(c b) a$. This they called the left invertive law. A groupoid $S$ is left almost semigroup ( abbreviated as LA-semigroup), if it satisfies the left invertive law: $(a b) c=(c b) a$. This structure is also known as Abel-Grassmann's groupoid ( abbreviated as AG-groupoid).

A groupoid $S$ is said to be medial ( resp. paramedial) if $(a b)(c d)=(a c)(b d)$ ( resp. $(a b)(c d)=(d b)(c a))$. In [12], an AG-groupoid is medial, but in general

2020 Mathematics Subject Classification: 03F55, 08A72, 20N25

Received: 13-07-2019, accepted: 13-04-2020. 
an AG-groupoid needs not be paramedial. Every AG-groupoid with left identity is paramedial, and also satisfies $a(b c)=b(a c),(a b)(c d)=(d c)(b a)$.

Algebraic structures play a prominent role in mathematics with wide ranging applications in many disciplines such as theoretical physics, computer sciences, control engineering, information sciences, coding theory, topological spaces and the like. Although semigroups concentrate on theoretical aspects, they also include applications in error-correcting codes, control engineering, formal language, computer science and information science. Algebraic structures especially ordered semigroups play a prominent role in mathematics with wide ranging applications in many disciplines such as control engineering, computer arithmetics, coding theory, sequential machines and formal languages. In [13], if $(S, \cdot, \leq)$ is an ordered semigroup and $\emptyset \neq A \subseteq S$, defined as follows: $(A]=\{s \in S: s \leq a$ for some $a \in A\}$. A nonempty subset $A$ of $S$ is a subsemigroup of $S$ if $A^{2} \subseteq A$. $A$ is a left ( resp. right) ideal of $S$ if following hold: (1) $S A \subseteq A$ (resp. $A S \subseteq A$ ). (2) If $a \in A$ and $b \in S$ such that $b \leq a$ implies $b \in A$ ( or $(A] \subseteq A$ ). A subsemigroup ( A non-empty subset) $A$ of $S$ is called a bi- ( generalized bi-) ideal of $S$ if (1) $A S A \subseteq A$. (2) If $a \in A$ and $b \in S$ such that $b \leq a$ implies $b \in A$. Every bi-ideal of $S$ is generalized bi-ideal of $S$. A subsemigroup $A$ of $S$ is called a $(1,2)$-ideal of $S$ if (1) $A S A^{2} \subseteq A$. (2) If $a \in A$ and $b \in S$ such that $b \leq a$ implies $b \in A$. In [13], an ordered semigroup $S$ is regular, if for every $a \in S$ there exists an element $x \in S$ such that $a \leq a x a$. Equivalent definitions are as follows: (1) $A \subseteq(A S A]$ for every $A \subseteq S$. (2) $a \in(a S a]$ for every $a \in S$. An ordered semigroup $S$ is $(2,2)$-regular, if for every $a \in S$ there exists an element $x \in S$ such that $a \leq a^{2} x a^{2}$. Equivalent definitions are as follows: (1) $A \subseteq\left(A^{2} S A^{2}\right]$ for every $A \subseteq S$. (2) $a \in\left(a^{2} S a^{2}\right]$ for every $a \in S$. In [13], an ordered semigroup $S$ is an intra-regular, if for every $a \in S$ there exist elements $x, y \in S$ such that $a \leq x a^{2} y$. Equivalent definitions are as follows: (1) $A \subseteq\left(S A^{2} S\right]$ for every $A \subseteq S$. (2) $a \in\left(S a^{2} S\right]$ for every $a \in S$. In this paper, we define the concept of anti fuzzy left ( resp. right, bi-, generalized bi-, $(1,2)$-) ideals in ordered AG-groupoids. We establish the study of regular ( resp. left regular, right regular, $(2,2)$-regular, left weakly regular, right weakly regular, intra-regular) ordered AG-groupoids in terms of anti fuzzy left ( resp. right, bi-, generalized bi-, $(1,2)$-) ideals. In this regard, we prove that in regular ( resp. left weakly regular) ordered AG-groupoids, the concept of anti fuzzy ( right, two-sided) ideals coincides. We also show that, in right regular ( resp. $(2,2)$-regular, right weakly regular, intra-regular) ordered AG-groupoids, the concept of anti fuzzy ( left, right, two-sided) ideals coincides. Also in left regular ordered AG-groupoids with left identity, the concept of anti fuzzy ( left, right, two-sided) ideals coincides. We also characterize the left weakly regular ordered AG-groupoids in terms of anti fuzzy right ( two-sided, bi-, generalized bi-) ideals.

\section{Fundamental Definitions and Results}

An ordered AG-groupoid $S$, is a partially ordered set, at the same time an AG-groupoid such that $a \leq b$, implies $a c \leq b c$ and $c a \leq c b$ for all $a, b, c \in S$. 
For $\emptyset \neq A \subseteq S$, we define a subset $(A]=\{s \in S: s \leq a$ for some $a \in A\}$ of $S$ and obviously $A \subseteq(A]$. If $A=\{a\}$, then we write (a] instead of $(\{a\}]$.

For $\emptyset \neq A \subseteq S$. $A$ is an AG-subgroupoid of $S$ if $A^{2} \subseteq A$. $A$ is a left ( resp. right) ideal of $S$ if (1) $S A \subseteq A$ ( resp. $A S \subseteq A$ ). (2) if $a \in A$ and $b \in S$ such that $b \leq a$ implies $b \in A$. Equivalent definition: $A$ is a left ( resp. right) ideal of $S$ if $(A] \subseteq A$ and $S A \subseteq A$ ( resp. $A S \subseteq A$ ). $A$ is an ideal of $S$, if $A$ is both a left and a right ideal of $S$.

An AG-subgroupoid $A$ of $S$ is a bi-ideal of $S$ if $(A S) A \subseteq A$ and $(A] \subseteq A$. A non empty subset $A$ of $S$ is a generalized bi-ideal of $S$ if $(A S) A \subseteq A$ and $(A] \subseteq A$. Every bi-ideal of $S$ is generalized bi-ideal of $S$. $(A] \subseteq A$.

An AG-subgroupoid $A$ of $S$ is a $(1,2)$-ideal of $S$ if $(A S) A^{2} \subseteq A$ and

Example 2.1. Let $S=\{a, b, c, d, e\}$. Define multiplication "” in $S$ as follows :

\begin{tabular}{l|lllll}
$\cdot$ & $a$ & $b$ & $c$ & $d$ & $e$ \\
\hline$a$ & $a$ & $a$ & $a$ & $a$ & $a$ \\
$b$ & $a$ & $a$ & $a$ & $a$ & $a$ \\
$c$ & $a$ & $a$ & $e$ & $c$ & $d$ \\
$d$ & $a$ & $a$ & $d$ & $e$ & $c$ \\
$e$ & $a$ & $a$ & $c$ & $d$ & $e$
\end{tabular}

and $\leq:=\{(a, a),(b, b),(c, c),(d, d),(e, e)\}$. Then $S$ is an ordered AG-groupoid. $A=\{c, d, e\}$ is an $A G$-subgroupoid of $S$ and $I=\{a, c, d, e\}$ is an ideal of $S$.

Every ideal ( whether left, right, two-sided) is an AG-subgroupoid but the converse is not true in general.

Now we give some important classes of ordered AG-groupoid.

An ordered AG-groupoid $S$ is regular if for every $a \in S$, there exists an element $x \in S$ such that $a \leq(a x) a$. Equivalent definitions are as follows:

(1) $A \subseteq((A S) A]$ for every $A \subseteq S$.

(2) $a \in((a S) a]$ for every $a \in S$.

An ordered AG-groupoid $S$ is left ( resp. right) regular, if for every $a \in S$, there exists an element $x \in S$ such that $a \leq x a^{2}$ ( resp. $a \leq a^{2} x$ ). Equivalent definitions are as follows:

(1) $A \subseteq\left(S A^{2}\right]$ ( resp. $\left.A \subseteq\left(A^{2} S\right]\right)$ for every $A \subseteq S$.

(2) $a \in\left(S a^{2}\right]$ ( resp. $\left.a \in\left(a^{2} S\right]\right)$ for every $a \in S$.

An ordered AG-groupoid $S$ is completely regular, if it is regular, left regular and right regular.

An ordered AG-groupoid $S$ is $(2,2)$-regular, if for every $a \in S$, there exists an element $x \in S$ such that $a \leq\left(a^{2} x\right) a^{2}$. Equivalent definitions are as follows:

(1) $A \subseteq\left(\left(A^{2} S\right) A^{2}\right]$ for every $A \subseteq S$.

(2) $a \in\left(\left(a^{2} S\right) a^{2}\right]$ for every $a \in S$. 
An ordered AG-groupoid $S$ is left ( resp. right) weakly regular, if for every $a \in S$, there exist elements $x, y \in S$ such that $a \leq(x a)(y a)$ (resp. $a \leq(a x)(a y)$ ). Equivalent definitions are as follows:

(1) $A \subseteq((S A)(S A)]$ ( resp. $A \subseteq((A S)(A S)])$ for every $A \subseteq S$.

(2) $a \in((S a)(S a)]$ ( resp. $a \in((a S)(a S)])$ for every $a \in S$.

An ordered AG-groupoid $S$ is intra-regular, if for every $a \in S$, there exist elements $x, y \in S$ such that $a \leq\left(x a^{2}\right) y$. Equivalent definitions are as follows:

(1) $A \subseteq\left(\left(S A^{2}\right) S\right]$ for every $A \subseteq S$.

(2) $a \in\left(\left(S a^{2}\right) S\right]$ for every $a \in S$.

We denote by $L(a), R(a), I(a)$ the left ideal, the right ideal and the ideal of $S$, respectively generated by $a$. We have $L(a)=\{s \in S: s \leq a$ or $s \leq x a$ for some $x \in S\}=(a \cup S a], R(a)=(a \cup a S], I(a)=(a \cup S a \cup a S \cup(S a) S]$.

An ordered AG-groupoid $S$ is locally associative if $(a . a) \cdot a=a .(a . a)$ for all $a \in S$.

Example 2.2. Let $S=\{a, b, c\}$ and define multiplication "” in $S$ as follows :

\begin{tabular}{l|lll}
$\cdot$ & $a$ & $b$ & $c$ \\
\hline$a$ & $c$ & $c$ & $b$ \\
$b$ & $b$ & $b$ & $b$ \\
$c$ & $b$ & $b$ & $b$
\end{tabular}

and $\leq:=\{(a, a),(b, b),(c, c)\}$. Then $(S, \cdot, \leq)$ is a locally associative ordered AG-groupoid.

In a locally associative ordered AG-groupoids $S$, we define powers of an element as follow: $a^{1}=a, a^{n+1}=a^{n} a$. If $S$ has a left identity $e$, we define $a^{0}=e$, as left identity is unique in an AG-groupoid. A locally associative ordered AG-groupoid $S$ with left identity $e$ has associative powers.

The notion of fuzzy set initiated by Zadeh in his classical paper [27] of 1965, which gives a natural framework for the generalizations of some basic notions of algebra, for example ( set, group, semigroup, ring, semiring, near-ring) theory, groupoids, real analysis, topology, differential equations and so forth. The study of fuzzy set in semigroups investigated by Kuroki [15]. He studied fuzzy ideals and fuzzy ( bi-, generalized bi-, quasi-, semiprime, semiprime quasi-) ideals of semigroups. A systematic exposition of fuzzy semigroups by Mordeson et. al appeared in [22], where one can find the theoretical results on fuzzy semigroups and their use in fuzzy finite state machines and languages. Fuzzy sets in ordered semigroups/ordered groupoids established by Kehayopulu et. al [14]. They also studied the concept of fuzzy ideals and fuzzy ( bi-, generalized bi-, quasi-) ideals in ordered semigroups [14].

Akram et al [1], studied the anti fuzzy left h-ideals in hemirings. Sardar et al [16], discussed the some properties of anti fuzzy subgroups. Kausar et al [26], initiated fuzzy bi-ideals on ordered AG-groupoids and also characterized the ordered AG-groupoids by using fuzzy bi-ideals. Kausar [3], characterized the ordered AG-groupoids by using the fuzzy ideals with thresholds $(\alpha, \beta]$. Kausar et al 
[8], established the anti fuzzy interior ideals on ordered AG-groupoids. Recently, Salahuddin et al [9], studied the characterizations of non-associative ordered semigroups in terms of intuitionistic fuzzy bi-ideals.

Shah et al [25], originated the studied of intuitionistic fuzzy normal LAsubrings over left almost-ring. Islam et al [6] initiated the intuitionistics fuzzy ideals with thresholds $(\alpha, \beta]$ in left almost ring. Javaid et al [5], also studied the left almost rings by fuzzy ideals. Waqar et al [2], studied the left almost rings by using the intuitionistic fuzzy bi-ideals. Kausar et al [4], explored the direct product of finite intuitionistic anti fuzzy normal LA-subrings over LA-rings. Waqar et al [7], investigated the direct product of finite fuzzy normal LA-subrings on Left Almostrings.

Kaviyarasu et al [11], studied the direct product of intuitionistic fuzzy KIdeals of INK-Algebras. Ahmad et al [17], initiated the m-Polar fuzzy ideals of BCK/BCI-algebras. Masarwah et al [18], reviewed the complete normality of m-pF subalgebras in BCK/BCI-algebras. Ahmad et al [19], evaluated the some properties of doubt bipolar fuzzy H-ideals in BCK/BCI-algebras. Masarwah et al [20], studied the doubt bipolar fuzzy subalgebras and ideals in BCK/BCI-algebras. Ahmad et al [21], determined the novel concepts of doubt bipolar fuzzy H-ideals of BCK/BCI-algebras. Hassan [23], approached toward a Q-neutrosophic soft set and its application in decision making. Qamar [24], studied the Q-neutrosophic soft relation and its application in decision making.

By a fuzzy subset $\mu$ of an ordered AG-groupoid $S$, we mean a function $\mu: S \rightarrow[0,1]$, the complement of $\mu$ is denoted by $\mu^{\prime}$ and defined as $\mu^{\prime}(x)=1-\mu(x)$ for all $x \in S$ is also a fuzzy subset of $S$.

A fuzzy subset $\mu$ of $S$ is an anti fuzzy AG-subgroupoid of $S$ if $\mu(x y) \leq$ $\max \{\mu(x), \mu(y)\}$ for all $x, y \in S . \mu$ is an anti fuzzy left ( resp. right) ideal of $S$ if $(1) \mu(x y) \leq \mu(y)(\operatorname{resp} . \mu(x y) \leq \mu(x))$, (2) $x \leq y$, implies $\mu(x) \leq \mu(y)$ for all $x, y \in S . \mu$ is an anti fuzzy ideal of $S$, if $\mu$ is both an anti fuzzy left and an anti fuzzy right ideal of $S$. Every anti fuzzy ideal ( whether left, right, two-sided) of $S$ is an anti fuzzy AG-subgroupoid of $S$ but the converse is not true in general.

An anti fuzzy AG-subgroupoid $\mu$ of $S$ is an anti fuzzy bi-ideal of $S$ if (1) $\mu((x a) y) \leq \max \{\mu(x), \mu(y)\},(2) \quad x \leq y$, implies $\mu(x) \leq \mu(y)$ for all $x, a, y \in S$. A fuzzy subset $\mu$ of $S$ is an anti fuzzy generalized bi-ideal of $S$ if $(1) \mu((x a) y) \leq$ $\max \{\mu(x), \mu(y)\},(2) x \leq y$, implies $\mu(x) \leq \mu(y)$ for all $x, a, y \in S$. Every anti fuzzy bi-ideal of $S$ is an anti fuzzy generalized bi-ideal of $S$. An anti fuzzy AG-subgroupoid $\mu$ of $S$ is an anti fuzzy $(1,2)$ - ideal of $S$ if $(1) \mu((x a)(y z)) \leq$ $\max \{\mu(x), \mu(y), \mu(z)\},(2) \quad x \leq y$, implies $\mu(x) \leq \mu(y)$ for all $x, a, y, z \in S$.

We denote by $F(S)$, the set of all fuzzy subsets of $S$. We define an order relation " $\subseteq$ " on $F(S)$ such that $f \subseteq g$ if and only if $f(x) \leq g(x)$ for all $x \in S$. Then $(F(S), \circ, \subseteq)$ is an ordered AG-groupoid.

By the symbols $\mu \wedge \gamma$ and $\mu \vee \gamma$, we mean the following fuzzy subsets $(\mu \wedge \gamma)(x)=\min \{\mu(x), \gamma(x)\}$ and $(\mu \vee \gamma)(x)=\max \{\mu(x), \gamma(x)\}$.

Let $a \in S$, we define a set $A_{a}=\{(y, z) \in S \times S \mid a \leq y z\}$. Let $\mu$ and $\gamma$ be two fuzzy subsets of $S$, then anti product of $\mu$ and $\gamma$ is denoted by $\mu \circ \gamma$ and 
defined as:

$$
\mu \circ \gamma(a)= \begin{cases}\wedge_{(y, z) \in A_{a}} \max \{\mu(y), \gamma(z)\} & \text { if } A_{a} \neq \emptyset \\ 1 & \text { if } A_{a}=\emptyset .\end{cases}
$$

Let $\emptyset \neq A \subseteq S$. Then the anti characteristic function of $A$ is denoted by $\chi_{A}^{C}$ and defined as

$$
\chi_{A}^{C}(a)=\left\{\begin{array}{c}
0 \text { if } a \in A \\
1 \text { if } a \notin A
\end{array}\right.
$$

An ordered AG-groupoid $S$ can be considered a fuzzy subset of itself and we write $S=\chi_{S}^{C}$, i.e., $S(x)=\chi_{S}^{C}(x)=0$ for all $x \in S$, this implies that $S(x)=0$ for all $x \in S$.

For $\emptyset \neq A, B \subseteq S, \quad A \subseteq B$ if and only if $\chi_{A}^{C} \geq \chi_{B}^{C}, \quad \chi_{A}^{C} \cap \chi_{B}^{C}=\chi_{A \cap B}^{C}$ and $\chi_{A}^{C} \circ \chi_{B}^{C}=\chi_{(A B]}^{C}$.

Let $\mu$ be a fuzzy subset of $S$, then for all $t \in(0,1]$, we define a set $L(\mu ; t)=\{x \in S \mid \mu(x) \leq t\}$, which is called a lower $t$-level of $\mu$. Now we discuss some fundamental crucial properties of ordered AG-groupoids, which will be very helpful for next section.

Lemma 2.3. Every anti fuzzy left ( resp. right, two-sided) ideal of an ordered $A G$-groupoid $S$ is an anti fuzzy bi-ideal of $S$.

Proof. Let $\mu$ be an anti fuzzy right ideal of $S$ and $x, a, y \in S$. Thus

$$
\begin{aligned}
\mu((x a) y) & \leq \mu(x a) \leq \mu(x) \\
\text { and } \mu((x a) y) & =\mu((y a) x) \leq \mu(y a) \leq \mu(y) . \\
& \Rightarrow \mu((x a) y) \leq \max \{\mu(x), \mu(y)\} .
\end{aligned}
$$

Hence $\mu$ is an anti fuzzy bi-ideal of $S$.

Lemma 2.4. Every anti fuzzy bi-ideal of an ordered AG-groupoid $S$ is an anti fuzzy $(1,2)$-ideal of $S$.

Proof. Suppose that $\mu$ is an anti fuzzy bi-ideal of $S$ and $a, x, y, z \in S$. Thus

$$
\mu((x a)(y z)) \leq \max \{\mu(x), \mu(y z)\} \leq \max \{\mu(x), \mu(y), \mu(z)\} .
$$

Therefore $\mu$ is an anti fuzzy $(1,2)$-ideal of $S$.

remark 2.5. Every anti fuzzy left ( right, two-sided) ideal of an ordered $A G$ groupoid $S$ is an anti fuzzy $(1,2)$-ideal of $S$.

Proposition 2.6. Let $S$ be an ordered AG-groupoid having the property $a \leq a^{2}$ for all $a \in S$. Then every anti fuzzy $(1,2)$-ideal of $S$ is an anti fuzzy bi-ideal of $S$.

Proof. Assume that $\mu$ is an anti fuzzy $(1,2)$-ideal of $S$ and $a, x, y \in S$. Now $(x a) y \leq(x a) y^{2}$, because $y \leq y^{2}$. Thus

$$
\begin{aligned}
\mu((x a) y) & \leq \mu\left((x a) y^{2}\right)=\mu((x a)(y y)) \\
& \leq \max \{\mu(x), \mu(y), \mu(y)\}=\max \{\mu(x), \mu(y)\} .
\end{aligned}
$$


So $\mu$ is an anti fuzzy bi-ideal of $S$.

remark 2.7. The concept of anti fuzzy ( bi-, (1,2)-) ideals coincides in ordered $A G$-groupoids $S$ having the property $a \leq a^{2}$ for all $a \in S$.

Example 2.8. Let $S=\{a, b, c, d\}$. Define multiplication " " in $S$ as follows :

\begin{tabular}{c|llll}
$\cdot$ & $a$ & $b$ & $c$ & $d$ \\
\hline$a$ & $c$ & $d$ & $a$ & $b$ \\
$b$ & $b$ & $c$ & $d$ & $a$ \\
$c$ & $a$ & $b$ & $c$ & $d$ \\
$d$ & $d$ & $a$ & $b$ & $c$
\end{tabular}

and $\leq:=\{(a, a),(b, b),(c, c),(d, d)\}$. Then $S$ is an ordered AG-groupoid. Let $\mu$ be a fuzzy subset of $S$. We define $\mu(a)=\mu(c)=0, \quad \mu(b)=\mu(d)=0.7$. Then $\mu$ is an anti fuzzy $A G$-subgroupoid of $S$.

Example 2.9. Let $S=\{a, b, c, d\}$. Define multiplication ". " in $S$ as follows :

\begin{tabular}{c|cccc}
$\cdot$ & $a$ & $b$ & $c$ & $d$ \\
\hline$a$ & $a$ & $a$ & $a$ & $a$ \\
$b$ & $a$ & $a$ & $a$ & $a$ \\
$c$ & $a$ & $a$ & $d$ & $a$ \\
$d$ & $a$ & $a$ & $c$ & $d$
\end{tabular}

and $\leq:=\{(a, a),(a, b),(b, a),(d, d)\}$. Then $S$ is an ordered AG-groupoid. Let $\mu$ be a fuzzy subset of $S$. We define $\mu(a)=\mu(c)=\mu(d)=0, \mu(b)=0.7$. Then $\mu$ is an anti fuzzy right ideal of $S . \mu$ is also an anti fuzzy bi- (resp. $(1,2)$-) ideal by Lemma 2.3 ( resp. 2.4).

Lemma 2.10. Let $S$ be an ordered $A G$-groupoid and $\emptyset \neq A \subseteq S$. Then the anti characteristic function $\chi_{(A]}^{C}$ of $(A]$ is a fuzzy subset of $S$ satisfying the condition $x \leq y \Rightarrow \chi_{(A]}^{C}(x) \leq \chi_{(A]}^{C}(y)$ for all $x, y \in S$.

Proposition 2.11. Let $S$ be an ordered $A G$-groupoid and $\emptyset \neq A \subseteq S$. Then $A=(A]$ if and only if the fuzzy subset $\chi_{A}^{C}$ of $S$ has the property $x \leq y \Rightarrow$ $\chi_{A}^{C}(x) \leq \chi_{A}^{C}(y)$ for all $x, y \in S$.

Lemma 2.12. Let $S$ be an ordered $A G$-groupoid and $\emptyset \neq A \subseteq S$. Then $A$ is an $A G$-subgroupoid ( resp. ( left, right, bi-, $(1,2)-$ ) ideal) of $S$ if and only if the anti characteristic function $\chi_{A}$ of $A$ is an anti fuzzy $A G$-subgroupoid ( resp. (left, right, bi-, $(1,2)-)$ ideal) of $S$.

Lemma 2.13. Let $\mu$ be a fuzzy subset of an ordered AG-groupoid S. Then $\mu$ is an anti fuzzy AG-subgroupoid (resp. ( left, right, bi-, (1,2)-) ideal) of $S$ if and only if upper t-level $U(\mu ; t)$ of $\mu$ is an AG-subgroupoid ( resp. (left, right, bi-, $(1,2)-)$ ideal $)$ of $S$ for all $t \in(0,1]$. 


\section{Anti Fuzzy Ideals On Ordered AG-Groupoids}

In this section, we extend the study of anti fuzzy ideals in ordered AGgroupoids and some results of characterizations of ordered AG-groupoids by using anti fuzzy left ( resp. right, bi-, generalized bi-) ideals.

Lemma 3.1. Every anti fuzzy right ideal of an ordered AG-groupoid $S$ with left identity $e$, is an anti fuzzy ideal of $S$.

Proof. Let $\mu$ be an anti fuzzy right ideal of $S$ and $x, y \in S$. Thus $\mu(x y)=$ $\mu((e x) y)=\mu((y x) e) \leq \mu(y x) \leq \mu(y)$. Hence $\mu$ is an anti fuzzy ideal of $S$.

remark 3.2. The concept of anti fuzzy ( right, two-sided) ideals coincides in ordered AG-groupoids with left identity.

Lemma 3.3. Let $S$ be an ordered AG-groupoid. Then $\mu \circ \gamma \supseteq \mu \cup \gamma$, for every anti fuzzy right ideal $\mu$ and every anti fuzzy left ideal $\gamma$ of $S$.

Proof. Let $\mu$ be an anti fuzzy right ideal and $\gamma$ be an anti fuzzy left ideal of $S$ and $x \in S$. If $A_{a}=\emptyset$, then obviously $\mu \circ \gamma \supseteq \mu \cup \gamma$, otherwise we have

$$
\begin{aligned}
(\mu \circ \gamma)(x) & =\wedge_{(y, z) \in A_{a}} \max \{\mu(y), \gamma(z)\} \\
& \geq \wedge_{(y, z) \in A_{a}} \max \{\mu(y z), \gamma(y z)\} \\
& =\wedge_{(y, z) \in A_{a}}\{\mu(y z) \vee \gamma(y z)\} \\
& =\wedge_{(y, z) \in A_{a}}\{(\mu \cup \gamma)(y z)\}=(\mu \cup \gamma)(x) . \\
& \Rightarrow \mu \circ \gamma \supseteq \mu \cup \gamma .
\end{aligned}
$$

Lemma 3.4. Every anti fuzzy right ideal of a regular ordered AG-groupoid $S$, is an anti fuzzy ideal of $S$.

Proof. Suppose that $\mu$ is an anti fuzzy right ideal of $S$ and $x, y \in S$, this implies that there exists $a \in S$ such that $x \leq(x a) x$. Thus $\mu(x y) \leq \mu(((x a) x) y)=$ $\mu((y x)(x a)) \leq \mu(y x) \leq \mu(y)$. Therefore $\mu$ is an anti fuzzy ideal of $S$.

remark 3.5. The concept of anti fuzzy ( right, two-sided) ideals coincides in regular ordered AG-groupoids.

Proposition 3.6. Let $S$ be a regular ordered AG-groupoid having the property $a \leq a^{2}$ for all $a \in S$, with left identity $e$. Then every anti fuzzy generalized bi-ideal of $S$ is an anti fuzzy bi-ideal of $S$.

Proof. Assume that $\mu$ is an anti fuzzy generalized bi-ideal of $S$ and $x, y \in S$, this means that there exists $a \in S$ such that $x \leq(x a) x$. We have to show that $\mu$ is an anti fuzzy AG-subgroupoid of $S$. Thus

$$
\begin{aligned}
\mu(x y) & \left.\leq \mu(((x a) x) y) \leq \mu\left(\left((x a) x^{2}\right) y\right)=\mu(((x a)(x x)) y)\right) \\
& =\mu((x((x a) x)) y) \leq \max \{\mu(x), \mu(y)\} .
\end{aligned}
$$

So $\mu$ is an anti fuzzy AG-subgroupoid of $S$. 
Lemma 3.7. Let $S$ be an odered AG-groupoid with left identity $e$ and $a \in S$. Then $S a$ is the smallest left ideal of $S$ containing a.

Proof. Let $x \in S a$ and $s \in S$, this implies that $x=s_{1} a, s_{1} \in S$. Thus

$$
\begin{aligned}
s x & =s\left(s_{1} a\right)=(e s)\left(s_{1} a\right)=\left(\left(s_{1} a\right) s\right) e=\left(\left(s_{1} a\right)(e s)\right) e \\
& =\left(\left(s_{1} e\right)(a s)\right) e=(e(a s))\left(s_{1} e\right)=(a s)\left(s_{1} e\right)=\left(\left(s_{1} e\right) s\right) a \in S a .
\end{aligned}
$$

Hence $s x \in S a$ and $(S a] \subseteq S a$. Now $a=e a \in S a$, so $S a$ is a left ideal of $S$ containing $a$. Let $I$ be another left ideal of $S$ containing $a$. Since $s a \in I$, because $I$ is a left ideal of $S$. But $s a \in S a$, this means that $S a \subseteq I$. Therefore $S a$ is a smallest left ideal of $S$ containing $a$.

Proposition 3.8. Let $S$ be an ordered AG-groupoid with left identity e and $a \in S$. Then $a S \cup S a$ is the smallest right ideal of $S$ containing $a$.

Proof. Let $x \in a S \cup S a$. We have to show that $(a S \cup S a) S \subseteq a S \cup S a$. Now

$$
\begin{aligned}
(a S \cup S a) S & =(a S) S \cup(S a) S=(S S) a \cup(S a)(e S) \\
& \subseteq S a \cup(S e)(a S)=S a \cup S(a S) \\
& =S a \cup a(S S) \subseteq S a \cup a S=a S \cup S a .
\end{aligned}
$$

Thus $(a S \cup S a) S \subseteq a S \cup S a$ and $(a S \cup S a] \subseteq \quad a S \cup S a$. Therefore $a S \cup S a$ is a right ideal of $S$. Since $a \in S a$, i.e., $a \in a S \cup S a$. Let $I$ be another right ideal of $S$ containing $a$. Now $a S \in I S \subseteq I$ and $S a=(S S) a=(a S) S \in(I S) S \subseteq I S \subseteq I$, i.e., $a S \cup S a \subseteq I$. Hence $a S \cup S a$ is a smallest right ideal of $S$ containing $a$.

Theorem 3.9. Let $S$ be an ordered AG-groupoid with left identity e, such that $(x e) S=x S$ for all $x \in S$. Then the following conditions are equivalent.

(1) $R$ is a regular.

(2) $\mu \cup \gamma=\mu \circ \gamma$ for every anti fuzzy right ideal $\mu$ and every anti fuzzy left ideal $\gamma$ of $S$.

Proof. Suppose that (1) holds. Since $\mu \cup \gamma \subseteq \mu \circ \gamma$, for every anti fuzzy right ideal $\mu$ and every anti fuzzy left ideal $\gamma$ of $S$ by the Lemma 3.3. Let $x \in S$, this implies that there exists an element $a \in S$ such that $x \leq(x a) x$. Thus

$$
\begin{aligned}
(\mu \circ \gamma)(x) & =\wedge_{(y, z) \in A_{a} \max \{\mu(y), \gamma(z)\}} \\
& \leq \max \{\mu(x a), \gamma(x)\} \leq \max \{\mu(x), \gamma(x)\} \\
& =(\mu \vee \gamma)(x)=(\mu \cup \gamma)(x) . \\
& \Rightarrow \mu \circ \gamma \subseteq \mu \cup \gamma .
\end{aligned}
$$

Hence $\mu \cup \gamma=\mu \circ \gamma$, i.e., (1) $\Rightarrow(2)$. Assume that (2) is true and $a \in S$. Then $S a$ is a left ideal of $S$ containing $a$ by the Lemma 3.7 and $a S \cup S a$ is a right ideal of $S$ containing $a$ by the Proposition 3.8. So $\chi_{S a}$ is an anti fuzzy left ideal and $\chi_{a S \cup S a}$ is an anti fuzzy right ideal of $S$, by the Lemma 2.12. By our assumption $\chi_{a S \cup S a} \cup \chi_{S a}=\chi_{a S \cup S a} \circ \chi_{S a}$, i.e., $\chi_{(a S \cup S a) \cup S a}=\chi_{((a S \cup S a) S a]}$. Thus $(a S \cup S a) \cup S a=((a S \cup S a) S a]$. Since $a \in(a S \cup S a) \cup S a$, i.e., $a \in((a S \cup S a) S a]$, 
so $a \in((a S)(S a) \cup(S a)(S a)]$. Now $(S a)(S a)=((S e) a)(S a)=((a e) S)(S a)=$ $(a S)(S a)$. This implies that

$$
((a S)(S a) \cup(S a)(S a)]=((a S)(S a) \cup(a S)(S a)]=((a S)(S a)] .
$$

Thus $a \in((a S)(S a)]$. Then

$$
\begin{aligned}
a & \leq(a x)(y a)=((y a) x) a=(((e y) a) x) a=(((a y) e) x) a \\
& =((x e)(a y)) a=(a((x e) y)) a \in(a S) a, \text { for any } x, y \in S .
\end{aligned}
$$

This means that $a \in((a S) a]$, i.e., $a$ is regular. Hence $S$ is a regular, i.e., $(2) \Rightarrow(1)$.

Theorem 3.10. Let $S$ be a regular locally associative ordered AG-groupoid having the property $a \leq a^{2}$ for all $a \in S$. Then for every anti fuzzy bi-ideal $\mu$ of $S$, $\mu\left(a^{n}\right)=\mu\left(a^{2 n}\right)$ for all $a \in S$, where $n$ is any positive integer.

Proof. For $n=1$. Let $a \in S$, this implies that there exists $x \in S$ such that $a \leq(a x) a$. Now $a \leq(a x) a \leq\left(a^{2} x\right) a^{2}, a \leq a^{2}$. Thus

$$
\begin{aligned}
\mu(a) & \leq \mu\left(\left(a^{2} x\right) a^{2}\right) \leq \max \left\{\mu\left(a^{2}\right), \mu\left(a^{2}\right)\right\}=\mu\left(a^{2}\right) \\
& =\mu(a a) \leq \max \{\mu(a), \mu(a)\}=\mu(a) . \\
& \Rightarrow \mu(a)=\mu\left(a^{2}\right) .
\end{aligned}
$$

Now $a^{2}=a a \leq\left(\left(a^{2} x\right) a^{2}\right)\left(\left(a^{2} x\right) a^{2}\right)=\left(a^{4} x^{2}\right) a^{4}$, then the result is true for $n=2$. Suppose that the result is true for $n=k$, i.e., $\mu\left(a^{k}\right)=\mu\left(a^{2 k}\right)$. Now $a^{k+1}=a^{k} a \leq\left(\left(a^{2 k} x^{k}\right) a^{2 k}\right)\left(\left(a^{2} x\right) a^{2}\right)=\left(a^{2(k+1)} x^{k+1}\right) a^{2(k+1)}$. Thus

$$
\begin{aligned}
\mu\left(a^{k+1}\right) & \leq \mu\left(\left(a^{2(k+1)} x^{k+1}\right) a^{2(k+1)}\right) \leq \max \left\{\mu\left(a^{2(k+1)}\right), \mu\left(a^{2(k+1)}\right)\right\} \\
& =\mu\left(a^{2(k+1)}\right)=\mu\left(a^{k+1} a^{k+1)}\right) \\
& \leq \max \left\{\mu\left(a^{k+1)}\right), \mu\left(a^{k+1)}\right)\right\}=\mu\left(a^{k+1)}\right) . \\
& \Rightarrow \mu\left(a^{k+1}\right)=\mu\left(a^{2(k+1)}\right) .
\end{aligned}
$$

Hence by induction method, the result is true for all positive integers.

Lemma 3.11. Every anti fuzzy left ( resp. right) ideal of $(2,2)$-regular ordered $A G$-groupoid $S$, is an anti fuzzy ideal of $S$.

Proof. Let $\mu$ be an anti fuzzy left ideal of $S$ and $x, y \in S$, this implies that there exists $a \in S$ such that $x \leq\left(x^{2} a\right) x^{2}$. Thus $\mu(x y) \leq \mu\left(\left(\left(x^{2} a\right) x^{2}\right) y\right)=\mu\left(\left(y x^{2}\right)\left(x^{2} a\right)\right) \leq$ $\mu((x x) a)=\mu((a x) x) \leq \mu(x)$. Hence $\mu$ is an anti fuzzy ideal of $S$.

remark 3.12. The concept of anti fuzzy (left, right, two-sided) ideals coincides in $(2,2)$-regular ordered AG-groupoids.

Proposition 3.13. Every anti fuzzy generalized bi-ideal of $(2,2)$-regular ordered $A G$-groupoid $S$ with left identity $e$, is an anti fuzzy bi-ideal of $S$. 
Proof. Suppose that $\mu$ is an anti fuzzy generalized bi-ideal of $S$ and $x, y \in S$, this means that there exists $a \in S$ such that $x \leq\left(x^{2} a\right) x^{2}$. We have to show that $\mu$ is an anti fuzzy AG-subgroupoid of $S$. Thus

$$
\begin{aligned}
\mu(x y) & \leq \mu\left(\left(\left(x^{2} a\right) x^{2}\right) y\right)=\mu\left(\left(\left(x^{2} a\right)(x x)\right) y\right) \\
& =\mu\left(\left(x\left(\left(x^{2} a\right) x\right)\right) y\right) \leq \max \{\mu(x), \mu(y)\} .
\end{aligned}
$$

Therefore $\mu$ is an anti fuzzy AG-subgroupoid of $S$.

Theorem 3.14. Let $S$ be a (2,2)-regular locally associative ordered AG-groupoid. Then for every anti fuzzy bi-ideal $\mu$ of $S, \mu\left(a^{n}\right)=\mu\left(a^{2 n}\right)$ for all $a \in S$, where $n$ is any positive integer.

Proof. Same as Theorem 3.10.

Lemma 3.15. Let $S$ be a right regular ordered AG-groupoid. Then every anti fuzzy left (resp. right) ideal of $S$ is an anti fuzzy ideal of $S$.

Proof. Let $\mu$ be an anti fuzzy left ideal of $S$ and $x, y \in S$, this implies that there exists $a \in S$ such that $x \leq x^{2} a$. Thus

$$
\begin{aligned}
\mu(x y) & \leq \mu\left(\left(x^{2} a\right) y\right)=\mu(((x x) a) y)=\mu(((a x) x) y) \\
& =\mu((y x)(a x)) \leq \mu(a x) \leq \mu(x) .
\end{aligned}
$$

Hence $\mu$ is an anti fuzzy ideal of $S$.

remark 3.16. The concept of anti fuzzy ( left, right, two-sided) ideals coincides in right regular ordered AG-groupoids.

Proposition 3.17. Every anti fuzzy generalized bi-ideal of a right regular ordered $A G$-groupoid $S$ with left identity $e$, is an anti fuzzy bi-ideal of $S$.

Proof. Suppose that $\mu$ is an anti fuzzy generalized bi-ideal of $S$ and $x, y \in S$, this means that there exists $a \in S$ such that $x \leq x^{2} a$. We have to show that $\mu$ is an anti fuzzy AG-subgroupoid of $S$. Thus

$$
\begin{aligned}
\mu(x y) & \leq \mu\left(\left(x^{2} a\right) y\right)=\mu(((x x)(e a)) y)=\mu(((a e)(x x)) y) \\
& =\mu((x((a e) x)) y) \leq \max \{\mu(x), \mu(y)\} .
\end{aligned}
$$

Therefore $\mu$ is an anti fuzzy AG-subgroupoid of $S$.

Theorem 3.18. Let $S$ be a right regular locally associative ordered AG-groupoid with left identity $e$. Then for every anti fuzzy right ideal $\mu$ of $S, \mu\left(a^{n}\right)=\mu\left(a^{2 n}\right)$ for all $a \in S$, where $n$ is any positive integer.

Proof. For $n=1$. Let $a \in S$, this means that there exists $x \in S$ such that $a \leq a^{2} x$. Thus

$$
\begin{aligned}
\mu(a) & \leq \mu\left(a^{2} x\right) \leq \mu\left(a^{2}\right)=\mu(a a) \\
& \leq \max \{\mu(a), \mu(a)\}=\mu(a) \\
& \Rightarrow \mu(a)=\mu\left(a^{2}\right)
\end{aligned}
$$


Now $a^{2}=a a \leq\left(a^{2} x\right)\left(a^{2} x\right)=a^{4} x^{2}$, then the result is true for $n=2$. Assume that the result is true for $n=k$, i.e., $\mu\left(a^{k}\right)=\mu\left(a^{2 k}\right)$. Now $a^{k+1}=a^{k} a \leq$ $\left(a^{2 k} x^{k}\right)\left(a^{2} x\right)=a^{2(k+1)} x^{k+1}$. Thus

$$
\begin{aligned}
\mu\left(a^{k+1}\right) & \leq \mu\left(a^{2(k+1)} x^{k+1}\right) \leq \mu\left(a^{2(k+1)}\right)=\mu\left(a^{k+1} a^{k+1}\right) \\
& \leq \max \left\{\mu\left(a^{k+1}\right), \mu\left(a^{k+1}\right)\right\}=\mu\left(a^{k+1}\right) . \\
& \Rightarrow \mu\left(a^{k+1}\right)=\mu\left(a^{2(k+1)}\right) .
\end{aligned}
$$

Therefore by induction method, the result is true for all positive integers.

Lemma 3.19. Let $S$ be a right regular locally associative ordered AG-groupoid with left identity $e$. Then for every anti fuzzy right ideal $\mu$ of $S, \mu(a b)=\mu(b a)$ for all $a, b \in S$.

Proof. Let $a, b \in S$. By using Theorem 3.18 ( for $n=1$ ). Now $\mu(a b)=\mu\left((a b)^{2}\right)=$ $\mu((a b)(a b))=\mu((b a)(b a))=\mu\left((b a)^{2}\right)=\mu(b a)$.

Theorem 3.20. Let $S$ be a regular and right regular locally associative ordered AG-groupoid. Then for every anti fuzzy right ideal $\mu$ of $S, \mu\left(a^{n}\right)=\mu\left(a^{3 n}\right)$ for all $a \in S$, where $n$ is any positive integer.

Proof. For $n=1$. Let $a \in S$, this implies that there exists $x \in S$ such that $a \leq(a x) a$ and $a \leq a^{2} x$. Now $a \leq(a x) a \leq(a x)\left(a^{2} x\right)=a^{3} x^{2}$. Thus

$$
\begin{aligned}
\mu(a) & \leq \mu\left(a^{3} x^{2}\right) \leq \mu\left(a^{3}\right)=\mu\left(a a^{2}\right) \leq \max \left\{\mu(a), \mu\left(a^{2}\right)\right\} \\
& \leq \max \{\mu(a), \mu(a), \mu(a)\}=\mu(a) . \\
& \Rightarrow \mu(a)=\mu\left(a^{3}\right) .
\end{aligned}
$$

Now $a^{2}=a a \leq\left(a^{3} x^{2}\right)\left(a^{3} x^{2}\right)=a^{6} x^{4}$, then the result is true for $n=2$. Suppose that the result is true for $n=k$, i.e., $\mu\left(a^{k}\right)=\mu\left(a^{3 k}\right)$. Now $a^{k+1}=a^{k} a \leq$ $\left(a^{3 k} x^{2 k}\right)\left(a^{3} x^{2}\right)=a^{3(k+1)} x^{2(k+1)}$. Thus

$$
\begin{aligned}
\mu\left(a^{k+1}\right) & \leq \mu\left(a^{3(k+1)} x^{2(k+1)}\right) \leq \mu\left(a^{3(k+1)}\right)=\mu\left(a^{3 k+3}\right) \\
& =\mu\left(a^{k+1} a^{2 k+2}\right) \leq \max \left\{\mu\left(a^{k+1}\right), \mu\left(a^{2 k+2}\right)\right\} \\
& \leq \max \left\{\mu\left(a^{k+1}\right), \mu\left(a^{k+1}\right), \mu\left(a^{k+1}\right)\right\}=\mu\left(a^{k+1}\right) . \\
& \Rightarrow \mu\left(a^{k+1}\right)=\mu\left(a^{3(k+1)}\right) .
\end{aligned}
$$

Hence by induction method, the result is true for all positive integers.

Lemma 3.21. Let $S$ be a left regular ordered AG-groupoid with left identity $e$. Then every anti fuzzy left ( resp. right) ideal of $S$ is an anti fuzzy ideal of $S$.

Proof. Let $\mu$ be an anti fuzzy left ideal of $S$ and $x, y \in S$, this implies that there exists $a \in S$ such that $x \leq a x^{2}$. Thus

$$
\begin{aligned}
\mu(x y) & \leq \mu\left(\left(a x^{2}\right) y\right)=\mu((a(x x)) y)=\mu((x(a x)) y) \\
& =\mu((y(a x)) x) \leq \mu((a x) x) \leq \mu(x) .
\end{aligned}
$$


Hence $\mu$ is an anti fuzzy ideal of $S$.

remark 3.22. The concept of anti fuzzy (left, right, two-sided) ideals coincides in left regular ordered AG-groupoids with left identity.

Proposition 3.23. Every anti fuzzy generalized bi-ideal of a left regular ordered $A G$-groupoid $S$ with left identity $e$, is an anti fuzzy bi-ideal of $S$.

Proof. Suppose that $\mu$ is an anti fuzzy generalized bi-ideal of $S$ and $x, y \in S$, this means that there exists $a \in S$ such that $x \leq a x^{2}$. We have to show that $\mu$ is an anti fuzzy AG-subgroupoid of $S$. Thus $\mu(x y) \leq \mu\left(\left(a x^{2}\right) y\right)=\mu((a(x x)) y)=$ $\mu((x(a x)) y) \leq \max \{\mu(x), \mu(y)\}$. Therefore $\mu$ is an anti fuzzy AG-subgroupoid of $S$.

remark 3.24. It is easy to see that, if $S$ is a left regular locally associative ordered AG-groupoid with left identity e. Then for every anti fuzzy left ideal $\mu$ of $S$, $\mu\left(a^{n}\right)=\mu\left(a^{2 n}\right)$ for all $a \in S$, where $n$ is any positive integer. And also for every anti fuzzy left ideal $\mu$ of $S, \mu(a b)=\mu(b a)$ for all $a, b \in S$.

Lemma 3.25. Let $S$ be a right weakly regular ordered AG-groupoid. Then every anti fuzzy left ( resp. right) ideal is an anti fuzzy ideal of $S$.

Proof. Let $\mu$ be an anti fuzzy left ideal of $S$ and $x, y \in S$, this implies that there exist $a, b \in S$ such that $x \leq(x a)(x b)$. Thus

$$
\begin{aligned}
\mu(x y) & \leq \mu(((x a)(x b)) y)=\mu((((x b) a) x) y) \\
& =\mu((((a b) x) x) y)=\mu((y x)((a b) x)) \\
& =\mu((y x)(n x)) \text { say } a b=n \\
& \leq \mu(n x) \leq \mu(x) .
\end{aligned}
$$

Hence $\mu$ is an anti fuzzy ideal of $S$.

remark 3.26. The concept of anti fuzzy (left, right, two-sided) ideals coincides in right weakly regular ordered AG-groupoids.

Proposition 3.27. Every anti fuzzy generalized bi-ideal of a right weakly regular ordered AG-groupoid $S$ with left identity e, is an anti fuzzy bi-ideal of $S$.

Proof. Suppose that $\mu$ is an anti fuzzy generalized bi-ideal of $S$ and $x, y \in S$, this means that there exist $a, b \in S$ such that $x \leq(x a)(x b)$. We have to show that $\mu$ is an anti fuzzy AG-subgroupoid of $S$. Thus $\mu(x y) \leq \mu(((x a)(x b)) y)=$ $\mu((x((x a) b)) y) \leq \max \{\mu(x), \mu(y)\}$. Therefore $\mu$ is an anti fuzzy AG-subgroupoid of $S$.

Lemma 3.28. Let $S$ be a left weakly regular ordered AG-groupoid. Then every anti fuzzy right ideal of $S$ is an anti fuzzy ideal of $S$.

Proof. Let $\mu$ be an anti fuzzy right ideal of $S$ and $x, y \in S$, this implies that there exist $a, b \in S$ such that $x \leq(a x)(b x)$. Thus

$$
\mu(x y) \leq \mu(((a x)(b x)) y)=\mu(y(b x))(a x) \leq \mu(y(b x)) \leq \mu(y) .
$$


Hence $\mu$ is an anti fuzzy ideal of $S$.

Lemma 3.29. Let $S$ be a left weakly regular ordered $A G$-groupoid with left identity e. Then every anti fuzzy left ideal of $S$ is an anti fuzzy ideal of $S$.

Proof. Suppose that $\mu$ is an anti fuzzy left ideal of $S$ and $x, y \in S$, this means that there exist $a, b \in S$ such that $x \leq(a x)(b x)$. Thus

$$
\begin{aligned}
\mu(x y) & \leq \mu(((a x)(b x)) y)=\mu(((a b)(x x)) y) \\
& =\mu((x((a b) x)) y)=\mu((y((a b) x)) x) \leq \mu(x) .
\end{aligned}
$$

Therefore $\mu$ is an anti fuzzy ideal of $S$.

Proposition 3.30. Every anti fuzzy generalized bi-ideal of a left weakly regular ordered AG-groupoid $S$ with left identity e, is an anti fuzzy bi-ideal of $S$.

Proof. Assume that $\mu$ is an anti fuzzy generalized bi-ideal of $S$ and $x, y \in S$, then there exist elements $a, b \in S$ such that $x \leq(a x)(b x)$. We have to show that $\mu$ is an anti fuzzy AG-subgroupoid of $S$. Thus

$$
\begin{aligned}
\mu(x y) & \leq \mu(((a x)(b x)) y)=\mu(((a b)(x x)) y) \\
& =\mu((x((a b) x)) y) \leq \min \{\mu(x), \mu(y)\} .
\end{aligned}
$$

So $\mu$ is an anti fuzzy AG-subgroupoid of $S$.

remark 3.31. It is easy to see that, if $S$ is a left (resp. right) weakly regular locally associative ordered AG-groupoid. Then for every anti fuzzy left ( resp. right) ideal $\mu$ of $S, \mu\left(a^{n}\right)=\mu\left(a^{2 n}\right)$ for all $a \in S$, where $n$ is any positive integer.

Theorem 3.32. Let $S$ be an ordered AG-groupoid with left identity e, such that $(x e) S=x S$ for all $x \in S$. Then the following conditions are equivalent.

(1) $S$ is a left weakly regular.

(2) $\mu \cup \gamma=\mu \circ \gamma$ for every anti fuzzy right ideal $\mu$ and every anti fuzzy left ideal $\gamma$ of $S$.

Proof. Suppose that (1) holds. Since $\mu \circ \gamma \supseteq \mu \cup \gamma$, for every anti fuzzy right ideal $\mu$ and every anti fuzzy left ideal $\gamma$ of $S$ by the Lemma 3.3. Let $x \in S$, this implies th at there exist elements $a, b \in S$ such that $x \leq(a x)(b x)=(a b)(x x)=x((a b) x)$. Thus

$$
\begin{aligned}
& (\mu \circ \gamma)(x)=\wedge_{(y, z) \in A_{a}} \max \{\mu(y), \gamma(z)\} \\
& \leq \max \{\mu(x), \gamma((a b) x)\} \leq \max \{\mu(x), \gamma(x)\} \\
& =(\mu \vee \gamma)(x)=(\mu \cup \gamma)(x) \text {. } \\
& \Rightarrow \mu \circ \gamma \subseteq \mu \cup \gamma \text {. }
\end{aligned}
$$

Hence $\mu \cup \gamma=\mu \circ \gamma$, i.e., (1) $\Rightarrow(2)$. Assume that (2) is true and $a \in S$. Then $S a$ is a left ideal of $S$ containing $a$ by the Lemma 3.7 and $a S \cup S a$ is a right ideal of $S$ containing $a$ by the Proposition 3.8. Therefore $\chi_{S a}$ is an anti fuzzy left ideal and $\chi_{a S \cup S a}$ is an anti fuzzy right ideal of $S$ by the Lemma 2.12. Then by our assumption $\chi_{a S \cup S a} \cup \chi_{S a}=\chi_{a S \cup S a} \circ \chi_{S a}$, i.e., $\chi_{(a S \cup S a) \cup S a}=\chi_{((a S \cup S a) S a]}$. Thus 
$(a S \cup S a) \cup S a=((a S \cup S a) S a]$. Since $a \in(a S \cup S a) \cup S a$, i.e., $a \in((a S \cup S a) S a]$, so $a \in((a S)(S a) \cup(S a)(S a)]$. Now

$$
\begin{aligned}
(a S)(S a) & =((e a)(S S))(S a)=((S S)(a e))(S a) \\
& =(((a e) S) S)(S a)=((a S) S)(S a) \\
& =((S S) a)(S a)=(S a)(S a) .
\end{aligned}
$$

This implies that $((a S)(S a) \cup(S a)(S a)]=((S a)(S a) \cup(S a)(S a)]=((S a)(S a)]$, thus $a \in((S a)(S a)]$. Hence $S$ is a left weakly regular, i.e., $(2) \Rightarrow(1)$.

Theorem 3.33. Let $S$ be an ordered AG-groupoid with left identity e, such that $(x e) S=x S$ for all $x \in S$. Then the following conditions are equivalent.

(1) $S$ is a left weakly regular. $\nu$ of $S$.

(2) $\mu \cup \nu \supseteq \mu \circ \nu$, for every anti fuzzy bi-ideal $\mu$ and every anti fuzzy ideal

(3) $\gamma \cup \nu \supseteq \gamma \circ \nu$, for every anti fuzzy generalized bi-ideal $\gamma$ and every anti fuzzy ideal $\nu$ of $S$.

Proof. Assume that (1) holds. Let $\gamma$ be an anti fuzzy generalized bi-ideal and $\nu$ be an anti fuzzy ideal of $S$. Let $x \in S$, this means that there exist elements $a, b \in S$ such that $x \leq(a x)(b x)=(a b)(x x)=x((a b) x)$. Thus

$$
\begin{aligned}
& (\gamma \circ \nu)(x)=\wedge_{(y, z) \in A_{a}} \max \{\gamma(y), \nu(z)\} \\
& \leq \min \{\gamma(x), \nu((a b) x)\} \leq \max \{\gamma(x), \nu(x)\} \\
& =(\gamma \vee \nu)(x)=(\gamma \cup \nu)(x) \text {. } \\
& \Rightarrow \gamma \cup \nu \supseteq \gamma \circ \nu \text {. }
\end{aligned}
$$

So $(1) \Rightarrow(3)$. It is clear that $(3) \Rightarrow(2)$. Suppose that (2) holds. Then $\mu \cup \nu \supseteq \mu \circ \nu$, where $\mu$ is an anti fuzzy right ideal of $S$. Since $\mu \circ \nu \supseteq \mu \cup \nu$, so $\mu \circ \nu=\mu \cup \nu$, Therefore $S$ is a left weakly regular by the Theorem 3.32, i.e., $(2) \Rightarrow(1)$.

Theorem 3.34. Let $S$ be an ordered AG-groupoid with left identity e, such that $(x e) S=x S$ for all $x \in S$. Then the following conditions are equivalent.

(1) $S$ is a left weakly regular.

(2) $\mu \cup \nu \cup \lambda \supseteq(\mu \circ \nu) \circ \lambda$, for every anti fuzzy bi-ideal $\mu$, every anti fuzzy ideal $\nu$ and every anti fuzzy right ideal $\lambda$ of $S$.

(3) $\gamma \cup \nu \cup \lambda \supseteq(\gamma \circ \nu) \circ \lambda$, for every anti fuzzy generalized bi-ideal $\gamma$, every anti fuzzy ideal $\nu$ and every anti fuzzy right ideal $\lambda$ of $S$.

Proof. Suppose that (1) holds. Let $\gamma$ be an anti fuzzy generalized bi-ideal, $\nu$ be an anti fuzzy ideal and $\lambda$ be an anti fuzzy right ideal of $S$. Let $x \in S$, then there exist elements $a, b \in S$ such that $x \leq(a x)(b x)$. Now

$$
\begin{aligned}
x & \leq(a x)(b x)=(x b)(x a) \\
x b & \leq((a x)(b x)) b=((x x)(b a)) b \\
& =(b(b a))(x x)=c(x x)=x(c x) \quad \text { say } c=b(b a)
\end{aligned}
$$


Thus

$$
\begin{aligned}
& ((\gamma \circ \nu) \circ \lambda)(x)=\wedge_{(y, z) \in A_{a}} \max \{(\gamma \circ \nu)(y), \lambda(z)\} \\
& \leq \max \{(\gamma \circ \nu)(x b), \lambda(x a)\} \\
& =\max \left\{\left(\wedge_{(s, t) \in A_{x b}} \max \{\gamma(s), \nu(t)\}\right), \lambda(x a)\right\} \\
& \leq \max \{(\max \{\gamma(x), \nu(c x)\}), \lambda(x a)\} \\
& \leq \max \{(\max \{\gamma(x), \nu(x)\}), \lambda(x)\} \\
& =\max \{\gamma(x), \nu(x), \lambda(x)\} \\
& =(\gamma \vee \nu \vee \lambda)(x)=(\gamma \cup \nu \cup \lambda)(x) \text {. } \\
& \Rightarrow \quad \gamma \cup \nu \cup \lambda \supseteq(\gamma \circ \nu) \circ \lambda \text {. }
\end{aligned}
$$

Hence $(1) \Rightarrow(3)$. It is clear that $(3) \Rightarrow(2)$, every anti fuzzy bi-ideal of $S$ is an anti fuzzy generalized bi-ideal of $S$. Assume that (2) is true. Then $\mu \cup \nu \cup S \supseteq(\mu \circ \nu) \circ S$, where $\mu$ is an anti fuzzy right ideal of $S$, i.e., $\mu \cup \nu \supseteq \mu \circ \nu$. Since $\mu \circ \nu \supseteq \mu \cup \nu$, so $\mu \circ \nu=\mu \cup \nu$. Therefore $S$ is a left weakly regular by the Theorem 3.32, i.e., $(2) \Rightarrow(1)$.

Lemma 3.35. Every anti fuzzy right ideal of an intra-regular ordered AG-groupoid $S$ is an anti fuzzy ideal of $S$.

Proof. Let $\mu$ be an anti fuzzy right ideal of $S$ and $x, y \in S$, this implies that there exist $a, b \in S$ such that $x \leq\left(a x^{2}\right) b$. Thus $\mu(x y) \leq \mu\left(\left(\left(a x^{2}\right) b\right) y\right)=\mu\left((y b)\left(a x^{2}\right)\right) \leq$ $\mu(y b) \leq \mu(y)$. Hence $\mu$ is an anti fuzzy ideal of $S$.

remark 3.36. The concept of anti fuzzy ( right, two-sided) ideals coincides in intra-regular ordered AG-groupoids.

Proposition 3.37. Every anti fuzzy generalized bi-ideal of an intra-regular ordered $A G$-groupoid $S$ with left identity $e$, is an anti fuzzy bi-ideal of $S$.

Proof. Suppose that $\mu$ is an anti fuzzy generalized bi-ideal of $S$ and $x, y \in S$, this means that there exist $a, b \in S$ such that $x \leq\left(a x^{2}\right) b$. We have to show that $\mu$ is an anti fuzzy AG-subgroupoid of $S$. Now

$$
\begin{aligned}
x & \leq\left(a x^{2}\right) b=\left(a x^{2}\right)(e b)=(a e)\left(x^{2} b\right)=(a e)((x x) b) \\
& =(a e)((b x) x)=(x(b x))(e a)=(x(b x)) a=(a(b x)) x \\
& =(a(b x))(e x)=(x e)((b x) a)=(b x)((x e) a)=(b x)((a e) x) \\
& =(x(a e))(x b)=x((x(a e)) b)=x n, \quad \text { say } n=(x(a e)) b
\end{aligned}
$$

Thus $\mu(x y) \leq \mu((x n) y) \leq \max \{\mu(x), \mu(y)\}$. Therefore $\mu$ is an anti fuzzy AG-subgroupoid of $S$.

Theorem 3.38. Let $S$ be an ordered AG-groupoid with left identity e, such that $(x e) S=x S$ for all $x \in S$. Then the following conditions are equivalent.

(1) $S$ is an intra-regular.

(2) $\mu \cup \gamma \supseteq \mu \circ \gamma$ for every anti fuzzy right ideal $\gamma$ and every anti fuzzy left ideal $\mu$ of $S$. 
Proof. Assume that (1) holds. Let $x \in S$, then there exist elements $a, b \in S$ such that $x \leq\left(a x^{2}\right) b$. Now

$$
\begin{aligned}
x & =\left(a x^{2}\right) b=(a(x x)) b=(x(a x))(e b) \\
& =(x e)((a x) b)=(a x)((x e) b) .
\end{aligned}
$$

Thus

$$
\begin{aligned}
& (\mu \circ \gamma)(x)=\wedge_{(y, z) \in A_{a}} \max \{\mu(y), \gamma(z)\} \\
& \leq \max \{\mu(a x), \gamma((x e) b)\} \leq \max \{\mu(x), \gamma(x)\} \\
& =(\mu \vee \gamma)(x)=(\mu \cup \gamma)(x) \text {. } \\
& \Rightarrow \mu \circ \gamma \subseteq \mu \cup \gamma \text {. }
\end{aligned}
$$

Hence $(1) \Rightarrow(2)$. Suppose that (2) is true. Let $a \in S$, then $S a$ is a left ideal of $S$ containing $a$ by the Lemma 3.7 and $a S \cup S a$ is a right ideal of $S$ containing $a$ by the Proposition 3.8. This means that $\chi_{S a}$ is an anti fuzzy left ideal and $\chi_{a S \cup S a}$ is an anti fuzzy right ideal of $S$, by the Lemma 2.12. By our supposition $\chi_{a S \cup S a} \cup \chi_{S a} \subseteq \chi_{S a} \circ \chi_{a S \cup S a}$, i.e., $\chi_{(a S \cup S a) \cup S a} \subseteq \chi_{((S a)(a S \cup S a)]}$. Thus $(a S \cup S a) \cup S a \subseteq(S a(a S \cup S a)]$. Since $a \in(a S \cup S a) \cup S a$, i.e., $a \in(S a(a S \cup S a)]=$ $((S a)(a S) \cup(S a)(S a)]$. Now

$$
\begin{aligned}
(S a)(a S) & =(S a)((e a)(S S))=(S a)((S S)(a e)) \\
& =(S a)(((a e) S) S)=(S a)((a S) S) \\
& =(S a)((S S) a)=(S a)(S a)
\end{aligned}
$$

This implies that

$$
\begin{aligned}
((S a)(a S) \cup(S a)(S a)] & =((S a)(S a) \cup(S a)(S a)] \\
& =((S a)(S a)]=((S a) a) S] \\
& =(((S a)(e a)) S]=(((S e)(a a)) S] \\
& =\left(\left(S a^{2}\right) S\right] .
\end{aligned}
$$

Thus $a \in\left(S a^{2}\right) S$, i.e., $a$ is an intra regular. Therefore $S$ is an intra-regular, i.e., $(2) \Rightarrow(1)$.

Theorem 3.39. Let $S$ be an intra-regular locally associative ordered AG-groupoid. Then for every anti fuzzy right ideal $\mu$ of $S, \mu\left(a^{n}\right)=\mu\left(a^{2 n}\right)$ for all $a \in S$, where $n$ is any positive integer.

Proof. For $n=1$. Let $a \in S$, this implies that there exist $x, y \in S$ such that $a \leq\left(x a^{2}\right) y$. Thus

$$
\begin{aligned}
\mu(a) & \leq \mu\left(\left(x a^{2}\right) y\right) \leq \mu\left(x a^{2}\right) \leq \mu\left(a^{2}\right) \\
& =\mu(a a) \leq \max \{\mu(a), \mu(a)\}=\mu(a) . \\
& \Rightarrow \mu(a)=\mu\left(a^{2}\right) .
\end{aligned}
$$

Now $a^{2}=a a \leq\left(\left(x a^{2}\right) y\right)\left(\left(x a^{2}\right) y\right)=\left(x^{2} a^{4}\right) y^{2}$, then the result is true for $n=2$. Suppose that the result is true for $n=k$, i.e., $\mu\left(a^{k}\right)=\mu\left(a^{2 k}\right)$. Now 


$$
\begin{aligned}
& a^{k+1}=a^{k} a \leq\left(\left(x^{k} a^{2 k}\right) y^{k}\right)\left(\left(x a^{2}\right) y\right)=\left(x^{k+1} a^{2(k+1)}\right) y^{k+1} \text {. Thus } \\
& \mu\left(a^{k+1}\right) \leq \mu\left(\left(x^{k+1} a^{2(k+1)}\right) y^{k+1}\right) \leq \mu\left(x^{k+1} a^{2(k+1)}\right) \\
& \leq \mu\left(a^{2(k+1)}\right)=\mu\left(a^{(k+1)} a^{(k+1)}\right) \\
& \leq \max \left\{\mu\left(a^{(k+1)}\right), \mu\left(a^{(k+1)}\right)\right\}=\mu\left(a^{(k+1)}\right) . \\
& \Rightarrow \mu\left(a^{k+1}\right)=\mu\left(a^{2(k+1)}\right) .
\end{aligned}
$$

Hence by induction method, the result is true for all positive integers.

Lemma 3.40. Let $S$ be an intra-regular locally associative ordered AG-groupoid with left identity $e$. Then for every anti fuzzy right ideal $\mu$ of $S, \mu(a b)=\mu(b a)$ for all $a, b \in S$.

Proof. Same as Lemma 3.19.

Proposition 3.41. Let $(S, \cdot \leq)$ be an ordered $A G$-groupoid with left identity $e$ such that

(1) $a \leq a^{2}$ for all $a \in S$.

(2) $a b \in((b a) S] \cap((S b) a]$ for all $a, b \in S$.

Then for every anti fuzzy bi-ideal $\mu$ of $S, \mu(a b)=\mu(b a)$ for all $a, b \in S$.

Proof. Since $a b \in((b a) S] \cap((S b) a]$, we have $a b \in((b a) S]$ and so $a b \leq(b a) x$ for some $x \in S$. Using (2), we get $(b a) x \in((x(b a)) S] \cap((S x)(b a)]$, this implies that $(b a) x \leq(y x)(b a)$ for some $y \in S$. Now

$$
\begin{aligned}
a b & \leq(b a) x \leq(b a)^{2} x=((b a)(b a)) x=((b a)(b a))(e x) \text { by }(1) \\
& =((b a) e)((b a) x) \leq((b a) e)((y x)(b a)) \\
& =((b a)(y x))(e(b a))=((b a)(y x))(b a)
\end{aligned}
$$

Thus $\mu(a b) \leq \mu((b a)(y x))(b a) \leq \max \{\mu(b a), \mu(b a)\}=\mu(b a)$. The reverse inequality is obvious. Hence $\mu(a b)=\mu(b a)$ for all $a, b \in S$.

\section{Conclusion}

Our purpose is to encourage the study and maturity of non associative algebraic structure (ordered AG-groupoid). The objective is to explain original methodological developments on ordered AG-groupoid, which will be very helpful for upcoming theory of algebraic structure. The ideal of fuzzy set is to characterize of ordered AG-groupoid are captivating a great attention of algebraist. The aim of this paper is to investigate, the study of ordered AG-groupoids by using the anti fuzzy left ( resp. right, bi-, generalized bi-, $(1,2)$-) ideals. 


\section{REFERENCES}

[1] Akram, M., and Dar, K.H., "On anti fuzzy left h-ideals in hemirings", Int. Math. Forum, 2(2007) 2295-2304.

[2] Kausar, N., and M. Waqar, M., "Characterizations of non-associative rings by their intuitionistic fuzzy bi-ideals", Eur. J. Pure Appl. Math., 121 (2019), 226-250.

[3] Kausar, N., "Characterizations of non-associative ordered semigroups by the properties of their fuzzy ideals with thresholds $(\alpha, \beta]$ ", Prikladnaya Diskretnaya Matematika., 43 (2019), 37-59.

[4] Kausar, N., "Direct product of finite intuitionistic anti fuzzy normal subrings over nonassociative rings", Eur. J. Pure Appl. Math., 122 (2019), 622-648.

[5] N. Kausar, N., Islam, B., Javaid, M., Amjad, S., and Ijaz, U., "Characterizations of nonassociative rings by the properties of their fuzzy ideals", Eur. J. Pure Appl. Math., 131 (2019), 820-833.

[6] Kausar, N., Islam, B., Amjad, S., and Waqar, M., "Intuitionistics fuzzy ideals with thresholds $(\alpha, \beta]$ in LA-rings",Eur. J. Pure Appl. Math., 123 (2019) 906-943.

7] Kausar, N., and Waqar, M., "Direct product of finite fuzzy normal subrings over nonassociative rings", Int. J. Anal. Appl., 175 (2019), 752-770.

[8] Kausar, N., Alesemi, M. and Salahuddin, "Anti fuzzy interior ideals on Ordered AGgroupoids", Eur. J. Pure Appl. Math., 131 (2020) 113-129.

[9] Kausar, N., Alesemi, M., Salahuddin, and Munir, M., "Characterizations of non-associative ordered semigroups by their intuitionistic fuzzy bi-ideals", Discontinuity, Nonlinearity, Complex. 92 (2020) 257-275.

[10] Kaviyarasu, M., Indhira, K., and Chandrasekaran, V.M., "Fuzzy sub algebras and Fuzzy K-ideals in INK-algebras", Int. J. Pure Appl. Math., 1136 (2017), 47-55.

[11] Kaviyarasu, M., Indhira, K., and Chandrasekaran, V.M., "Direct Product of Intuitionistic Fuzzy K-Ideals of INK-Algebras", Materials Today: Proceedings, 16 (2019) 449-455.

[12] Kazim, M.A., and Naseerudin, M., "On almost semigroups", Aligarh Bull. Math., 2 (1972), 1-7.

[13] Kehayopulu, N., "On intra-regular ordered semigroups", Semigroup Forum, 46 (1993), 271278.

[14] Kehayopulu, N., and Tsingelis, M., "Fuzzy sets in ordered groupoids", Semigroup Forum, $65(2002)$ 128-132.

[15] Kuroki, N., "On fuzzy semigroups", Inform. Sci., 5 (1991), 203-36.

[16] Majumder, S.K., and Sardar, S.K., "On some properties of anti fuzzy subgroups", J. Mech. Cont. Math. Sci., 3 (2008), 337-343.

[17] Al-Masarwah, A., and Ahmad, A.G., "m-Polar fuzzy ideals of BCK/BCI-algebras", J. King Saud Univ.-Sci., 314 (2019), 1220-1226.

[18] Al-Masarwah, A., and Ahmad, A.G., "On (complete) normality of m-pF subalgebras in BCK/BCI-algebras", AIMS Mathematics, 43 (2019), 740-750.

[19] Al-Masarwah, A., and Ahmad, A.G., "On some properties of doubt bipolar fuzzy H-ideals in BCK/BCI-algebras", Eur. J. Pure Appl. Math., 113 (2018), 652-670.

[20] Al-Masarwah, A., and Ahmad, A.G., "Doubt bipolar fuzzy subalgebras and ideals in BCK/BCI- algebras", J. Math. Anal., 93 (2018), 9-27.

[21] Al-Masarwah, A., and Ahmad, A.G., "Novel concepts of doubt bipolar fuzzy H-ideals of BCK/BCI-algebras", Int. J. Innov. Comput. Inf. Control., 146 (2018), 2025-2041.

[22] Mordeson, J. N., Malik D.S., and Kuroki, N., Fuzzy semigroups, Springer, Berlin, 2003.

[23] Abu Qamar, M., and Hassan, N., "An approach toward a Q-neutrosophic soft set and its application in decision making", Symmetry, 112 (2019), 139.

[24] Abu Qamar, M., and Hassan, N., "Q-neutrosophic soft relation and its application in decision making", Entropy, 203, (2018), 172.

[25] Shah, T., Kausar, N., and Rehman, I., "Intuitionistic fuzzy normal subrings over a nonassociative ring", An. St. Univ. Ovidius Constanta, 20 (2012), 369-386. 
[26] Shah, T., and Kausar, N., "Characterizations of non-associative ordered semigroups by their fuzzy bi-ideals", Theor. Comput. Sci., 529 (2014), 96-110.

[27] Zadeh, L.A., "Fuzzy sets", Inf. Control, 8 (1965), 338-363. 